\title{
Role of Chemohormonal Therapy in Management of Patients with Metastatic Hormone Sensitive Prostate Cancer
}

\author{
Sanjay Hinduja1, Adnan Khan ${ }^{2}$, Uzair B. Chaudhary ${ }^{3 *}$ \\ ${ }^{1}$ Department of Internal Medicine, University of California San Francisco-Fresno, Fresno, CA, USA \\ ${ }^{2}$ Wayne State University School of Medicine, Detroit, MI, USA \\ ${ }^{3}$ Division of Hematology/Oncology, University of California San Francisco-Fresno, Fresno, CA, USA \\ Email: *uchaudhary@fresno.ucsf.edu
}

How to cite this paper: Hinduja, S., Khan, A. and Chaudhary, U.B. (2017) Role of Chemohormonal Therapy in Management of Patients with Metastatic Hormone Sensitive Prostate Cancer. Journal of Cancer Therapy, 8, 1149-1159.

https://doi.org/10.4236/jct.2017.813098

Received: May 12, 2017

Accepted: December 10, 2017

Published: December 13, 2017

Copyright $\odot 2017$ by authors and Scientific Research Publishing Inc. This work is licensed under the Creative Commons Attribution International License (CC BY 4.0).

http://creativecommons.org/licenses/by/4.0/

\begin{abstract}
Androgen deprivation therapy has been the mainstay of treatment for management of metastatic hormone sensitive prostate cancer. Recently conducted randomized controlled trials with docetaxel based chemotherapy have shed light on benefit of early use chemotherapy for hormone sensitive prostate cancer. In this review, we aim to comprehensively describe recent trials which are changing the paradigm in support of up front use of docetaxel based chemotherapy for metastatic hormone sensitive prostate cancer.
\end{abstract}

\section{Keywords}

Metastatic Prostate Cancer, Hormone Sensitive Disease

\section{Introduction}

Prostate cancer is the third leading cause of cancer death among American men. The American cancer society estimates that there will be up to 161,000 newly diagnosed cases along with 26,000 prostate cancer deaths in 2017 [1]. There has been an increase in diagnosis of prostate cancer especially in the US, largely due to increased number of biopsies being performed on asymptomatic men with elevated Prostate specific antigen (PSA). Nearly 50\% of men will develop rising PSA after local therapy for prostate cancer and most will eventually develop metastatic disease.

\section{Management of Hormone Sensitive Prostate Cancer}

Huggins and Hodges introduced the idea of androgen dependence in 1972 [2]. 
Androgen deprivation therapy (ADT) has been a long mainstay of first line therapy for prostate cancer. Prostate cancer is primarily driven by androgens produced in the testes. Androgen production is regulated by hypothalamic pituitary axis. Testosterone produced by testes accounts for upto $90 \%-95 \%$ of circulating testosterone. The adrenal glands also produce androgens which get converted peripherally to testosterone. This is physiologically insignificant compared to testosterone released from testes [3]. Androgen deprivation can be established by either surgical orchiectomy or medical orchiectomy. Medical orchiectomy is established by way of gonadotropin releasing hormone ( $\mathrm{GnRh}$ ) agonists such as leuprolide which suppress leutenizing hormone (LH) and leads to decreased testosterone production. Testosterone production can also be decreased by way of GnRH antagonist such as degarelix which bind to GnRH receptors on pituitary gonadotropin-producing cells and leading to decreased testosterone production.

\section{Evolution to Hormone Resistant Prostate Cancer}

Despite new developments in androgen deprivation therapy; eventually nearly all patients will develop hormone resistant prostate cancer [4] [5]. It is typically defined as rising PSA with low levels of testosterone with or without presence of metastatic disease. Several mechanisms have been identified which lead to hormone resistance. These are broadly classified into androgen receptor mediated mechanisms and androgen receptor independent mechanisms.

One well studied mechanism of androgen receptor reactivation in hormone resistant prostate cancer is via increased expression of androgen receptor. The frequency of androgen receptor over expression and gene amplification has been reported to be low at baseline, but increases significantly when evaluated in the ADT resistant population [6]. This is due to genomic amplification of the androgen receptor locus or up regulation of androgen receptor protein levels. Up to $30 \%$ of hormone resistant prostate cancer tumors harbor androgen gene receptor amplification in comparison to none for hormone sensitive prostate cancer. This enables the hormone resistant prostate cancer cells to be hypersensitive to low levels of androgens leading to disease progression [7]. This highlights the importance of continued androgen signaling in driving cell growth in hormone resistant prostate cancer.

Recent autopsy studies and tissue biopsy studies have demonstrated high levels of androgens in tumor tissue of men with hormone resistant prostate cancer [8] [9]. In the absence of circulating androgen production by testes; hormone resistant tumor cells convert weak androgens to potent androgens such as dihydrotestosterone (DHT). They also have de novo production of androgens from cholesterol within the tumor tissue itself. This leads to increased levels of testosterone within tumor cells therefore driving cancer cell growth [3] [10].

Somatic androgen receptor mutations which occur in response to androgen deprivation have been identified. A review of 27 clinical studies found that an- 
drogen receptor mutations in androgen dependent tumors ranged from $2 \%$ $25 \%$, while the incidence in hormone resistant tumors was slightly higher at $10 \%$ $40 \%$ [11]. Some androgen receptor mutations cause gain of function in androgen receptors leading to hypersensitivity to circulating androgens. Two well-known mutations are AR-T877A and AR-W741C, which convert the antiandrogens flutamide and bicalutamide to partial agonists, respectively [4].

Androgen receptor splice variants have recently been studied in their role for contributing to hormone resistant prostate cancer. The exact mechanism of androgen receptor splice variants is disputed however it is postulated that it acts via reaction of the normal androgen receptor transcriptome or generating an altered androgen receptor gene signature [4].

Sustained androgen receptor blockade contributes to the selection of prostate cancer cell clones which are able to upregulate a bypass pathway which leads to hormone resistance [4]. Alterations in various oncogenic pathways including MAPK,RB1, PI3K, AKT, mTOR seen in advanced prostate cancer which promote survival, proliferation, cell cycle progression, and conversely regulate androgen receptor signaling through various feedback mechanisms have been identified [12].

\section{Management of Hormone Resistant Prostate Cancer}

Significant advances have been made in the management for hormone resistant prostate cancer over the last decade. Androgen synthesis inhibitors, androgen receptor antagonists, radiopharmaceuticals, immunotherapy and advanced chemotherapy have revolutionized the management of castrate resistant prostate cancer. Abiraterone irreversibly binds to 17 alpha carboxylase in doing so it blocks the synthesis of testosterone in testes, tumor and adrenals. In two phase III trials abiraterone plus prednisone prolonged overall survival compared to prednisone alone in patients who were previously treated with chemotherapy and in those who were chemotherapy naïve [13]. Enzalutamide blocks the binding of androgen to receptor leading to decreased effect of testosterone. In a phase III trial enzalutamide showed prolonged overall survival compared to placebo [14]. Immunotherapy has also been used for treatment for hormone resistant prostate cancer. One such therapy is Sipuleucel-T which is a form of dendritic cell vaccine. In a phase III clinical trial Sipuleucel-T showed improvement in overall survival however did not show disease regression [15]. Sipuleucel-T is an option for men with castration-resistant metastatic prostate cancer who are asymptomatic or have minimally symptomatic disease. Radium 223 an alpha particle emitting agent radio pharmaceutical which has been utilized for skeletal metastasis in the absence of visceral metastasis. In a phase III clinical trial it showed improved overall survival as well as decrease incidence of skeletal events in men with painful bony metastasis [16].

Docetaxel based chemotherapy has long been established as upfront chemotherapy for hormone resistance prostate cancer. In 2004 a prospective phase III 
clinical trial conducted by Petrylak et al. compared mitoxantrone and prednisone with docetaxel and estramustine in patients with metastatic hormone resistant prostate cancer. This trial showed that the group which received docetaxel and estramustine had a longer median time of progression compared with mitoxantrone and prednisone [17].

In another randomized phase III clinical trial conducted by Tannock et all also showed efficacy of docetaxel in the treatment for metastatic hormone resistant prostate cancer. This trial compared docetaxel (weekly and 3 weekly dosing) with mitoxantrone-prednisone. The median survival was 16.5 months in the mitoxantrone-prednisone and 18.9 months in the docetaxel (every 3 week dosing) and 17.4 months in docetaxel weekly dosing group [18]. With these trials docetaxel given every 3 weeks was the first cytotoxic agent approved for prostate cancer which showed improvement in overall survival.

Cabazitaxel another taxane based chemotherapy agent has been studied in androgen independent prostate cancer. Cabazitaxel was compared to mitoxantrone in a phase III clinical trial in patients who had progressed on docetaxel. This trial showed that men treated with cabazitaxel plus prednisone had an increased overall survival and progression free survival compared to those who were treated with mitoxantrone plus prednisone [19]. Data from another phase III clinical trial showed that cabazitaxel had greater tumor response rate when compared to docetaxel however there no noted increase in progression free survival between the two groups [20].

\section{Rationale for Upfront Chemotherapy in Metastatic Hormone Sensitive Prostate Cancer}

Conventionally taxane based chemotherapy was reserved for metastatic hormone resistant prostate cancer. With the advent of newer therapies focus shifted towards sequence of use of these therapies. There was a paucity of data on timing of the use of chemotherapy. With the use of newer therapies taxane based chemotherapy was pushed even further down. Recently conducted trials have shown that taxane based chemotherapy up-front alongside ADT has shown improvements in overall survival in patients with hormone-sensitive metastatic prostate cancer. Three large clinical trials, (GEUG-AFU 15), (CHAARTED), and (STAMPEDE) have recently been done to study the effects of docetaxel used with $\mathrm{ADT}$ as treatment for hormone-sensitive disease. These clinical trials GETUGAFU15, CHAARTED, and STAMPEDE have changed the paradigm for chemotherapy use in metastatic hormone sensitive prostate cancer. We will discuss these trials in detail below.

\section{GETUG-AFU 15 Trial}

In this study 385 patients received treatment for metastatic hormone resistant prostate cancer [21]. One hundred ninety-three patients received androgen deprivation therapy alone and 192 received ADT with up to nine cycles of docetaxel 
chemotherapy. The patients were classified into three groups: good prognosis, intermediate prognosis, and poor prognosis.

The primary endpoint studied was overall survival. In addition, the study looked at clinical progression-free survival (cPFS) and biochemical progressionfree survival (bPFS). In the ADT alone arm, the median overall survival was 54.2 months compared to 58.9 months in the ADT and docetaxel arm. Median bPFS was 12.9 months in the ADT alone group and 22.9 months in the ADT and docetaxel group. Finally, median cPFS was 15.4 months in the ADT alone arm and 23.5 months in the ADT and docetaxel arm.

In the combination arm, 39 patients (21\%) stopped treatment early due to toxicity. Approximately a third (61 patients) experience neutropenia of grade 3 5 and 25 patients suffered from febrile neutropenia of grade 3 - 5. Overall, a total of 72 severe adverse events occurred in the ADT plus docetaxel group, with the most common ones being neutropenia, febrile neutropenia, and abnormal liver function tests. None of these events occurred in the ADT alone group, however, patients did suffer from adverse events like anemia, hot flushes, decreased libido, and erectile dysfunction.

This study showed that the combination of ADT with docetaxel did not show a significant improvement in overall survival in patients suffering from metastatic hormone resistant prostate cancer. Improvements seen in bPFS and cPFS without remarkable results in the primary endpoint point towards not using docetaxel as a first line therapy for such patients.

There are, however, a number of limitations in this study that may have contributed to the minimal overall survival difference between the two groups. First, the small sample size of 385 patients may have led to erroneous results. Second, the median overall survival of 54.2 months in the ADT alone arm was much higher than is usually seen for patients with metastatic hormone resistant prostate cancer. Third, although the patients were separated into three prognosis groups, specific information regarding the disease level of the patients in each group was not specified. Finally, 62\% (120 patients) of the patients that were receiving $\mathrm{ADT}$ alone also received docetaxel at the time of disease progression from hormone sensitive to hormone resistant prostate cancer. This additional treatment could have minimized the gap in overall survival between the two groups.

\section{CHAARTED Trial}

In the CHAARTED study, a total of 790 patients underwent treatment for metastatic, hormone sensitive prostate cancer [22]. Of the 790 patients, 393 were given $\mathrm{ADT}$ alone and 397 were provided the combination of ADT plus six cycles of docetaxel. Patients were classified according to the extent of their metastases into high-volume and low-volume disease states, with $65 \%$ of the patients exhibiting the former.

The primary endpoints of this study were: median overall survival, PSA levels 
after completion of treatment, time to the development of castration-resistant prostate cancer, and time to clinical progression. This study showed improvement in median overall survival by 13.6 months in combination therapy (ADT plus docetaxel) group. This was further pronounced in patients with high volume disease where the median overall was 17.0 months longer in combination group compared with ADT alone group. The proportion of patients that saw their PSA levels drop to below $0.2 \mathrm{ng} / \mathrm{mL}$ at 12 months of treatment was $16.8 \%$ in the $\mathrm{ADT}$ alone group and $27.7 \%$ in the combination group. The time to development of hormone resistant cancer was 11.7 months in the ADT alone group and 20.2 months in the combination group. Lastly, time to clinical progression was also improved in combination group which was noted to be 33.0 months compared with ADT alone group which was 19.8 months.

There were multiple adverse events present in the group that received Docetaxel along with ADT. $6 \%$ of these patients experienced neutropenic fever, and $2 \%$ had a grade 3 or 4 infection with neutropenia. Additionally, $2 \%$ of the combination group patients had a treatment-related grade 3 or 4 allergic reaction, and approximately $1 \%$ suffered from a thromboembolic event.

This study showed that combination therapy using docetaxel for metastatic, hormone-sensitive prostate cancer resulted in more promising outcomes for men than ADT alone. These patients saw a longer overall survival, a higher rate of decrease in PSA levels, and a longer period of time before clinical progression and the development of hormone resistant disease. The results were significantly more pronounced for men with high-volume of disease as compared to those with low-volume.

\section{STAMPEDE Trial}

In this study, a total of 2962 patients were enrolled and treated for hormonesensitive prostate cancer [23]. This study further subdivided patients into highrisk locally advanced, metastatic, and node positive. High-risk locally advanced was defined as having any two of the following characteristics: Gleason score of 8 - 10, prostate specific antigen greater than or equal to $40 \mathrm{ng} / \mathrm{ml}$, and Stage T3/ T4. There were four treatment arms which are as follows: standard of care (SOC) and zoledronic acid (ZA) and docetaxel (doc) (593 patients), standard of care and docetaxel (592 patients), standard of care and zoledronic acid (593 patients), standard of care only (1184 patients). Standard of care was defined as hormone therapy for minimum of two years with gonadotropin-releasing hormone agonists or antagonists or for patients with non metastatic diseases oral anti androgens alone.

The outcomes measured in this study were overall survival and failure-free survival. In the SOC only arm, median overall survival was 71 months and median failure-free survival was 20 months. In the SOC + ZA arm, median overall survival was not reached and median failure-free survival was 22 months. There were significant improvements seen in both overall survival and failure-free survival 
in the arms that included docetaxel. In the SOC + Doc arm, median survival was 81 months and median failure-free survival was 37 months. Finally, in the SOC + $\mathrm{ZA}+$ Doc arm, the median survival was 76 months and the median failure-free survival was 36 months.

The number of individuals that suffered from skeletal-related events also improved in the population that was receiving docetaxel treatment. In the SOC only arm, 328 patients suffered from at least one skeletal-related event, compared to 112 patients and 108 patients in the SOC + Doc arm and the SOC + ZA + Doc arm, respectively. No improvement was seen in the SOC + ZA arm, with 153 patients suffering from at least one skeletal-related event. Mean time to a skeletal-related event was 61.4 months in the SOC only arm, 68.0 months in the SOC + Doc arm, and 68.3 months in the SOC + ZA + Doc arm. This shows the increased mean time to first skeletal even in the docetaxel group.

A downside to the docetaxel treatment was that a greater proportion of these patients suffered from clinically significant toxicity of grade 3 or higher. The breakdown for the four groups was as follows: $17 \%$ for SOC group, $39 \%$ for SOC $+\mathrm{ZA}+$ DOC group, $36 \%$ for the SOC + Doc group, $15 \%$ for the SOC $+\mathrm{ZA}$ group.

This study affirmed that docetaxel therapy showed evidence of survival improvement if given in the beginning with long-term hormone therapy in patients suffering from metastatic or high-risk non-metastatic prostate cancer, while zoledronic acid did not. Patients receiving docetaxel saw a longer overall survival and failure-free survival compared to patients not receiving docetaxel, and less of them suffered from skeletal-related events. However, a higher number of these patients experienced adverse events as compared to patients receiving standard of care only or standard of care with zoledronic acid. This study established that docetaxel should be included as part of the first-line therapy for prostate cancer, while zoledronic acid should not.

\section{Discussion}

These three studies show that addition of docetaxel to androgen-deprivation therapy helped improve overall survival in patients suffering from metastatic hormone-sensitive prostate cancer. In the GETUG-AFU-15 trial, the earliest of the three studies, there was no significant benefit shown in patients receiving docetaxel with ADT as compared to those receiving ADT alone. These results, however, are skewed based on the limitations discussed earlier. On the other hand, the CHAARTED trial showed that patients receiving docetaxel with ADT had a convincingly longer overall survival (OS), with these patients living on average 13.6 months longer than those receiving ADT alone. The STAMPEDE trial showed similar results, with patients receiving docetaxel showing an overall survival increase of 10 months compared to their standard-of-care (SOC) only counterparts.

A previous meta-analysis done on these three trials showed similar improve- 
ments [24]. For each of the studies, the hazard ratio (HR) was calculated for the overall survival, with the HR of less than one suggesting a benefit for the patients that received the combined treatment. The use of docetaxel with ADT showed an OS benefit for patients with metastatic and non-metastatic cancer, with a HR of 0.74 . When looking at just metastatic patients, the HR was 0.73 , again showing an overall improvement. These results were mirrored in a pooled analysis that studied 10 randomized clinical trials assessing treatment methods for patients with metastatic and high-risk localized hormone-sensitive prostate cancer, showing a HR for OS of 0.75 for metastatic hormone-sensitive disease patients [25].

The effects of docetaxel were more pronounced in patients suffering from high-volume disease. In the CHAARTED trial, high volume diseases was determined as presence of visceral metastases or four or more bone lesions, with one or more of the these bone lesions beyond the vertebral bodies and pelvis. When focusing on these patients, this trial showed an overall survival improvement of 17.0 months in those receiving docetaxel along with ADT. The previous metaanalysis saw fairly limited statistical difference in results when comparing highvolume to low-volume disease patients [23]. In this comparison, patients from the GETUG-AFU-15 and CHAARTED trials showed an insignificant change in the OS as the hazard ratio for high-volume disease patients was 0.67 and that for low-volume disease patients was 0.80 . As such, the meta-analysis did not find a relationship between disease volume and treatment efficacy.

It is important to discuss the toxicity associated with docetaxel, which was present in all three trials. In the combination-arm of the GETUG-AFU-15 trial, approximately a third of the patients experienced neutropenia of grade 3 - 5 and 25 patients suffered from febrile neutropenia of grade $3-5$. In the CHAARTED trial, $6 \%$ of the combination group experienced neutropenic fever, and $2 \%$ had a grade 3 - 4 infection with neutropenia. In the STAMPEDE trial, 36\% of the SOC plus docetaxel patients suffered from significant toxicity of grade 3 or higher compared to $17 \%$ in the SOC-only arm. The meta-analysis and pooled analysis both saw an increased number of adverse events associated with the combined therapy arms, with the most common toxicity being febrile neutropenia, based on the pooled analysis [21] [22]. In spite of these results, the considerable improvement in overall survival for these patients proves the validity of docetaxel as a first-line therapy.

The number of cycles of docetaxel given to the patients varied amongst the three trials. The GETUG-AFU-15 trial gave $75 \mathrm{mg} / \mathrm{m}^{2}$ IV docetaxel once every three weeks for up to nine cycles. The CHAARTED and STAMPEDE trials both gave $75 \mathrm{mg} / \mathrm{m}^{2}$ IV docetaxel once every three weeks for up to six cycles. In the GETUG-AFU-15 trial, 21\% of patients were unable to finish their treatment of docetaxel due to toxic effects. Therefore, since both the CHAARTED and STAMPEDE trials showed promising results, six cycles of docetaxel are recommended to avoid the adverse effects associated with further cycles. 


\section{Future Directions}

Six cycles of docetaxel based chemotherapy in combination with hormonal therapy have clearly shown overall survival benefit in patients with high risk metastatic hormone sensitive prostate cancer. Given development of newer therapeutic modalities, it will be important to design combination strategies with docetaxel based chemotherapy to further improve outcome. One such strategy is combining docetaxel with RAD-223 which will be studied through Alliance co-operative group in the near future. One recently published study shows the upfront use of abiraterone had improved outcomes in a population similar to the aforementioned clinical trials [26]. Continued emphasis on development of bio-markers, genomic signature and incorporation of new agents will continue to improve outcome for patients with high risk metastatic hormone sensitive prostate cancer.

\section{References}

[1] Siegel, R.L., Miller, K.D. and Jemal, A. (2017) Cancer Statistics, 2017. CA: A Cancer Journal for Clinicians, 67, 7-30. https://doi.org/10.3322/caac.21387

[2] Huggins, C. and Hodges, C.V. (1972) Studies on Prostatic Cancer. I. The Effect of castration, of estrogen and androgen injection on serum phosphatases in metastatic carcinoma of the prostate. CA: A Cancer Journal for Clinicians, 22, 232-240. https://doi.org/10.3322/canjclin.22.4.232

[3] Nussey, S. and Whitehead, S. (2001) Endocrinology: An Integrated Approach. BIOS Scientific Publishers BIOS Scientific Publishers Limited, Oxford. https://doi.org/10.4324/9780203450437

[4] Karantanos, T., Corn, P.G. and Thompson, T.C. (2013) Prostate Cancer Progression after Androgen Deprivation Therapy: Mechanisms of Castrate Resistance and Novel Therapeutic Approaches. Oncogene, 32, 5501-5511. https://doi.org/10.1038/onc.2013.206

[5] Attar, R.M., Takimoto, C.H. and Gottardis, M.M. (2009) Castration-Resistant Prostate Cancer: Locking up the Molecular Escape Routes. Clinical Cancer Research, 15, 3251-3255. https://doi.org/10.1158/1078-0432.CCR-08-1171

[6] Maughan, B.L. and Antonarakis, E.S. (2015) Androgen Pathway Resistance in Prostate Cancer and Therapeutic Implications. Expert Opinion on Pharmacotherapy, 16, 1521-1537. https://doi.org/10.1517/14656566.2015.1055249

[7] Hoang, D.T., et al. (2017) Androgen Receptor-Dependent and -Independent Mechanisms Driving Prostate Cancer Progression: Opportunities for Therapeutic Targeting from Multiple Angles. Oncotarget, 8, 3724-3745.

[8] Montgomery, R.B., et al. (2008) Maintenance of Intratumoral Androgens in Metastatic Prostate Cancer: A Mechanism for Castration-Resistant Tumor Growth. Cancer Research, 68, 4447-4454. https://doi.org/10.1158/0008-5472.CAN-08-0249

[9] Mohler, J.L., et al. (2004) The Androgen Axis in Recurrent Prostate Cancer. Clinical Cancer Research, 10, 440-448. https://doi.org/10.1158/1078-0432.CCR-1146-03

[10] Harris, W.P., et al. (2009) Androgen Deprivation Therapy: Progress in Understanding Mechanisms of Resistance and Optimizing Androgen Depletion. Nature Clinical Practice Urology, 6, 76-85. https://doi.org/10.1038/ncpuro1296 
[11] Koochekpour, S. (2010) Androgen Receptor Signaling and Mutations in Prostate Cancer. Asian Journal of Andrology, 12, 639-657. https://doi.org/10.1038/aja.2010.89

[12] Hernandez-Aya, L.F. and Hussain, M. (2015) Why Chemotherapy Should Be Given Early for Men with Metastatic Prostate Cancer. American Society of Clinical Oncology Educational Book, e263-e269. https://doi.org/10.14694/EdBook_AM.2015.35.e263

[13] Rathkopf, D.E., et al. (2013) Updated Interim Analysis (IA) of COU-AA-302, a Randomized Phase III study of Abiraterone Acetate (AA) in Patients (pts) with Metastatic Castration-Resistant Prostate Cancer (mCRPC) without Prior Chemotherapy. Journal of Clinical Oncology, 31, 5.

[14] Scher, H.I., et al. (2012) Increased Survival with Enzalutamide in Prostate Cancer after Chemotherapy. The New England Journal of Medicine, 367, 1187-1197. https://doi.org/10.1056/NEJMoa1207506

[15] Kantoff, P.W., et al. (2010) Sipuleucel-T Immunotherapy for Castration-Resistant Prostate Cancer. The New England Journal of Medicine, 363, 411-422. https://doi.org/10.1056/NEJMoa1001294

[16] Parker, C., et al. (2013) Alpha Emitter Radium-223 and Survival in Metastatic Prostate Cancer. The New England Journal of Medicine, 369, 213-223. https://doi.org/10.1056/NEJMoa1213755

[17] Petrylak, D.P., et al. (2004) Docetaxel and Estramustine Compared with Mitoxantrone and Prednisone for Advanced Refractory Prostate Cancer. The New England Journal of Medicine, 351, 1513-1520. https://doi.org/10.1056/NEJMoa041318

[18] Tannock, I.F., et al. (2004) Docetaxel plus Prednisone or Mitoxantrone plus Prednisone for Advanced Prostate Cancer. The New England Journal of Medicine, 351, 1502-1512. https://doi.org/10.1056/NEJMoa040720

[19] Eigl, B.J., et al. (2005) Timing Is Everything: Preclinical Evidence Supporting Simultaneous Rather than Sequential Chemohormonal Therapy for Prostate Cancer. Clinical Cancer Research, 11, 4905-4911. https://doi.org/10.1158/1078-0432.CCR-04-2140

[20] Sartor, A.O. (2016) Cabazitaxel vs Docetaxel in Chemotherapy-Naive (CN) Patients with Metastatic Castration-Resistant Prostate Cancer (mCRPC): A Three-Arm Phase III Study (FIRSTANA). Journal of Clinical Oncology, 34.

[21] Gravis, G., et al. (2013) Androgen-Deprivation Therapy Alone or with Docetaxel in Non-Castrate Metastatic Prostate Cancer (GETUG-AFU 15): A Randomised, OpenLabel, Phase 3 Trial. The Lancet Oncology, 14, 149-158. https://doi.org/10.1016/S1470-2045(12)70560-0

[22] Sweeney, C.J., et al. (2015) Chemohormonal Therapy in Metastatic Hormone-Sensitive Prostate Cancer. New England Journal of Medicine, 373, 737-746. https://doi.org/10.1056/NEJMoa1503747

[23] James, N.D., et al. (2016) Addition of Docetaxel, Zoledronic Acid, or Both to First-Line Long-Term Hormone Therapy in Prostate Cancer (STAMPEDE): Survival Results from an Adaptive, Multiarm, Multistage, Platform Randomised Controlled Trial. The Lancet, 387, 1163-1177. https://doi.org/10.1016/S0140-6736(15)01037-5

[24] Tucci, M., et al. (2016) Addition of Docetaxel to Androgen Deprivation Therapy for Patients with Hormone-Sensitive Metastatic Prostate Cancer: A Systematic Review and Meta-Analysis. European Urology, 69, 563-573.

https://doi.org/10.1016/j.eururo.2015.09.013 
[25] Abdel-Rahman, O. (2016) Combined Chemohormonal Strategy in Hormone-Sensitive Prostate Cancer: A Pooled Analysis of Randomized Studies. Clinical Genitourinary Cancer, 14, 203-209. https://doi.org/10.1016/j.clgc.2015.12.004

[26] Fizazi, K., et al. (2017) Abiraterone plus Prednisone in Metastatic, Castration-Sensitive Prostate Cancer. New England Journal of Medicine, 377, 352-360.

https://doi.org/10.1056/NEJMoa1704174 